\title{
A Laser-Sharp View of Electron Correlations
}

\section{A high-resolution photoemission experiment provides an unprecedented test of a theory describing the effects of strong electron correlations in solids.}

\section{by Fu-chun Zhang*}

$\mathrm{T}$ he photoelectric effect refers to the emission of electrons from a metal that is injected with light-a phenomenon that was discovered over 100 years ago and explained by Einstein. Today, the effect is the basis for a powerful experimental method known as angle-resolved photoemission spectroscopy (ARPES). This technique uses light to take a "picture" of a material's electronic energy bands, the structure of which dictates many material properties. Researchers have steadily increased the resolution of these electron pictures by various means, including employing lasers as the light source. Now, using laser-based ARPES, Anna Tamai of the University of Geneva and colleagues provide an unprecedented test of a theory for materials in which electron correlations are strong [1]. The researchers studied the unconventional superconductor,
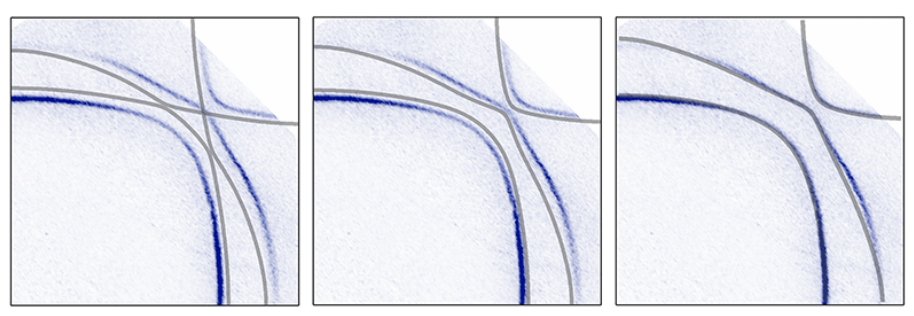

Figure 1: Three comparisons between the electronic energy bands (blue) measured by Tamai et al. and

density-functional-theory calculations (grey). The calculations correspond to no spin-orbit coupling (left); spin-orbit coupling (center); and spin-orbit coupling that is enhanced by electron correlations (right). (A. Tamai et al. [1])

\footnotetext{
* Kavli Institute for Theoretical Sciences, University of Chinese Academy of Sciences, Beijing, China
}

$\mathrm{Sr}_{2} \mathrm{RuO}_{4}$, and determined that correlations enhance a parameter known as spin-orbit coupling (SOC) by a factor of 2 -in agreement with the theoretical prediction. Their accurate measurement of SOC may also help physicists resolve a puzzle surrounding the superconducting state of $\mathrm{Sr}_{2} \mathrm{RuO}_{4}$.

$\mathrm{Sr}_{2} \mathrm{RuO}_{4}$ has been a font of interesting physics [2]. In 1994, experimentalists discovered that the material becomes superconducting at about $1.5 \mathrm{~K}$. Theorists soon speculated that $\mathrm{Sr}_{2} \mathrm{RuO}_{4}$ was unlike other known superconductors. They also conjectured that its "Cooper pairs" of electrons, which carry the superconducting current, had a spin of 1 instead of a spin of 0 [3], indicating an unusual pairing mechanism. But the type of pairing has been an ongoing subject of debate. Support for the spin-1 picture comes from various early experiments, such as measurements of the superconducting phase, muon spin rotation, and the Kerr effect, whereas a recent nuclear magnetic resonance (NMR) experiment indicates spin-0 pairs [4]. The nature of the pairing is also relevant to the possibility that $\mathrm{Sr}_{2} \mathrm{RuO}_{4}$ is a topological superconductor, an exotic phase of interest for a robust form of quantum computing.

$\mathrm{Sr}_{2} \mathrm{RuO}_{4}$ is also attractive because its physics, including the pairing mechanism, is affected by interactions (or "correlations") between the electrons. In fact, the material has become a model system for understanding these effects both experimentally and theoretically. A theory developed specifically for materials with strong correlations, known as dynamical mean-field theory (DMFT), predicts that electrons in $\mathrm{Sr}_{2} \mathrm{RuO}_{4}$ enhance the coupling between electron momentum and spin (spin-orbit coupling) [5, 6]. But this predicted enhancement has yet to be tested.

The work by Tamai and co-workers provides the best such test to date [1]. The team investigated electron correlations in $\mathrm{Sr}_{2} \mathrm{RuO}_{4}$ using ARPES to measure three energy bands near the Fermi energy. These bands are derived from three of the $4 d$ orbitals of the ruthenium atoms, and their qualitative shape has been measured in previous ARPES experiments. What was harder to see until now was a theoretically predicted separation (in energy and momentum) between the bands. This band "splitting" occurs at the Fermi energy, and it is caused by SOC involving the $4 d$ electrons.

The team determined the Fermi surface and the energy 
bands of $\mathrm{Sr}_{2} \mathrm{RuO}_{4}$ with unprecedented accuracy by using an 11-eV laser light source with an energy resolution of 3 $\mathrm{meV}$ and an angular resolution of $0.2^{\circ}$. Compared with earlier ARPES studies, the bands measured by Tamai et al. have narrower widths, making it easier to see the distortions induced by SOC. The group also took steps to suppress contributions from surface states, ensuring that their measured energy bands correspond to "bulk" electrons. (The experiments were performed at $5 \mathrm{~K}$ in the "normal" state of $\mathrm{Sr}_{2} \mathrm{RuO}_{4}$.)

The team determined the magnitude of the correlationenhanced SOC in $\mathrm{Sr}_{2} \mathrm{RuO}_{4}$ experimentally by measuring the band splitting. The enhanced SOC is about twice as large as its "bare" value (no correlations), in agreement with the value calculated within DMFT. A separate, direct measurement of the correlation effects comes from comparing the measured bands with three calculations based on densityfunctional theory (DFT). This computational tool is more standard than DMFT, but it typically applies to materials without strong electron correlations. DFT calculations were performed without SOC, with bare SOC, and with an "effective" SOC that includes an enhancement from electron correlations (Fig. 1). The excellent agreement of the calculation (Fig. 1, right) with the ARPES data provides direct measurement of the enhanced SOC. These tests of DFT and DMFT give weight to the applicability of these approaches to $\mathrm{Sr}_{2} \mathrm{RuO}_{4}$ as well as to other materials with multiple $d$-electron orbitals, strong SOC, and strong electron correlations, such as the iron-based superconductors.

The "cleanliness" of the ARPES data also allowed the authors to confirm a fundamental assumption of DMFT that is related to the determination of so-called electron selfenergies. These are shifts in energy that result from electron interactions, and they can have sizable effects on the energy bands. DMFT typically assumes the self-energies are momentum independent to simplify calculations. The researchers confirmed this "ansatz" by extracting self-energies from their measured bands, a result that provides further strength to the applicability of DMFT for $\mathrm{Sr}_{2} \mathrm{RuO}_{4}$.

Beyond testing theory, knowing the strength of the SOC is of interest for understanding superconductivity in $\mathrm{Sr}_{2} \mathrm{RuO}_{4}$ - a far from settled topic. Strong SOC could substantially mix the spin- 0 and spin- 1 states of the Cooper pairs. Figuring out whether the SOC is sufficiently strong for this mixing to occur will require additional calculations [7]. But this step is worth making as physicists try to reconcile the new NMR data [4], which suggest spin-0 Cooper pairs, with older measurements, which support spin-1 pairs [2].

This research is published in Physical Review X.

\section{REFERENCES}

[1] A. Tamai et al., "High-resolution photoemission on $\mathrm{Sr}_{2} \mathrm{RuO}_{4}$ reveals correlation-enhanced effective spin-orbit coupling and dominantly local self-energies," Phys. Rev. X 9, 021048 (2019).

[2] A. P. Mackenzie and Y. Maeno, "The superconductivity of $\mathrm{Sr}_{2} \mathrm{RuO}_{4}$ and the physics of spin-triplet pairing," Rev. Mod. Phys. 75, 657 (2003).

[3] T. M. Rice and M. Sigrist, " $\mathrm{Sr}_{2} \mathrm{RuO}_{4}$ : An electronic analogue of ${ }^{3}$ He?" J. Phys. Condens. Matter 7, L643 (1995).

[4] A. Pustogow et al., "Pronounced drop of ${ }^{17} \mathrm{O}$ NMR Knight shift in superconducting state of $\mathrm{Sr}_{2} \mathrm{RuO}_{4}$," arXiv:1904.00047.

[5] G. Zhang et al., "Fermi Surface of $\mathrm{Sr}_{2} \mathrm{RuO}_{4}$ : Spin-orbit and anisotropic Coulomb interaction effects," Phys. Rev. Lett. 116, 106402 (2016).

[6] M. Kim et al., "Spin-orbit coupling and electronic correlations in $\mathrm{Sr}_{2} \mathrm{RuO}_{4}$," Phys. Rev. Lett. 120, 126401 (2018).

[7] Q. H. Wang, C. Platt, Y. Yang, C. Honerkamp, F. C. Zhang, W. Hanke, T. M. Rice, and R. Thomale, "Theory of superconductivity in a three-orbital model of $\mathrm{Sr}_{2} \mathrm{RuO}_{4}$," Europhys. Lett. 104, 17013 (2013).

10.1103/Physics.12.89 